\section{Scalp Myiasis in an Ulcerated Abscess in a 7-year Old Child}

\section{Sir,}

Myiasis refers to a group of disorders in which living vertebrates are infested with dipterous larvae (two-winged maggots). ${ }^{1}$ This is a rare human disease and occurs more commonly, although not exclusively, in tropical climates. Besides poor hygiene and inaccessible sanitation, local conditions like leprosy and dermatitis, as well as general systemic illnesses like diabetes, malignancy, and psychiatric illnesses provide hotbed for myiasis. ${ }^{1,2}$

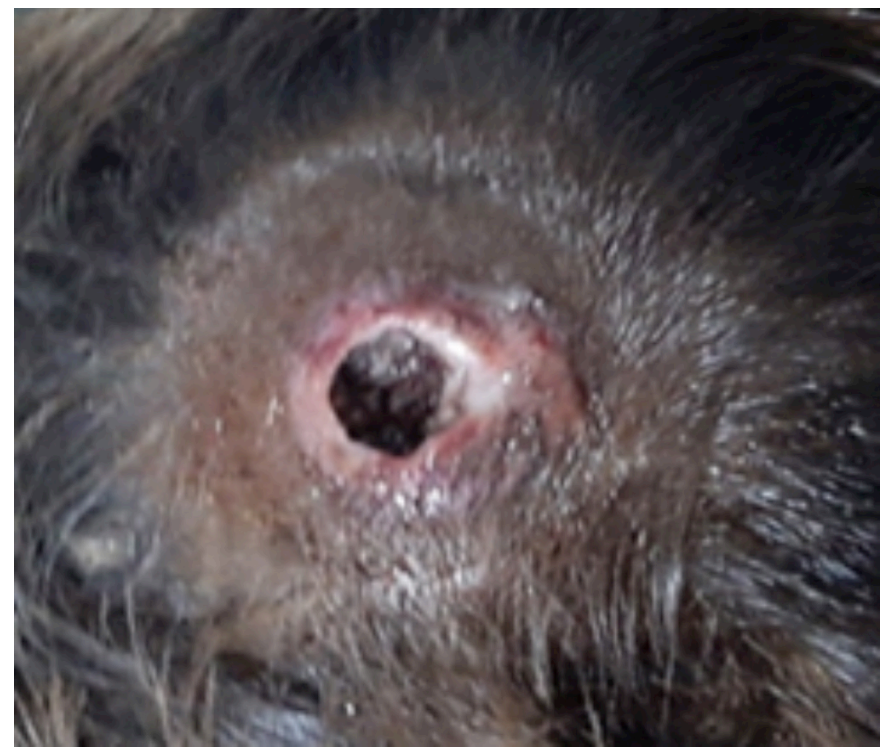

Figure 1a: Crater-shaped abscess with necrotic edges.

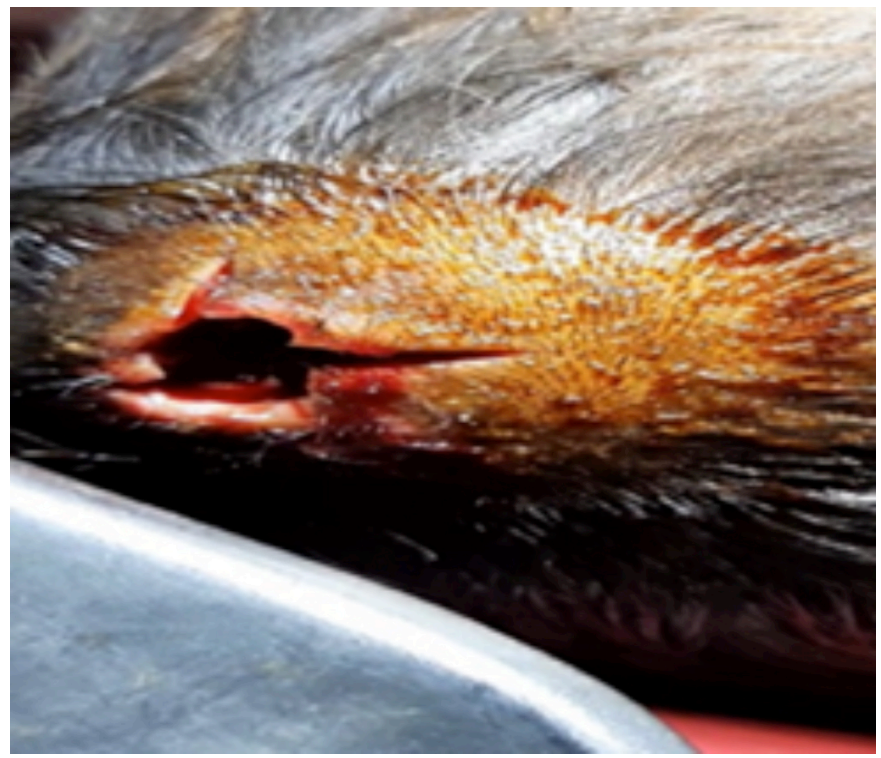

Figure 1b: Excision of margins and cruciate shaped incision.

A seven-year boy presented to Neurosurgical Emergency with a deeply ulcerated scalp abscess. Guardian of the patient gave history of appearance of a minor boil on the scalp two weeks ago, which later ulcerated spontaneously, producing a thick viscid pus with a deeply pungent smell. They were homeless and were living in a brick kiln for last seven months with no access to clean water and proper sanitation.

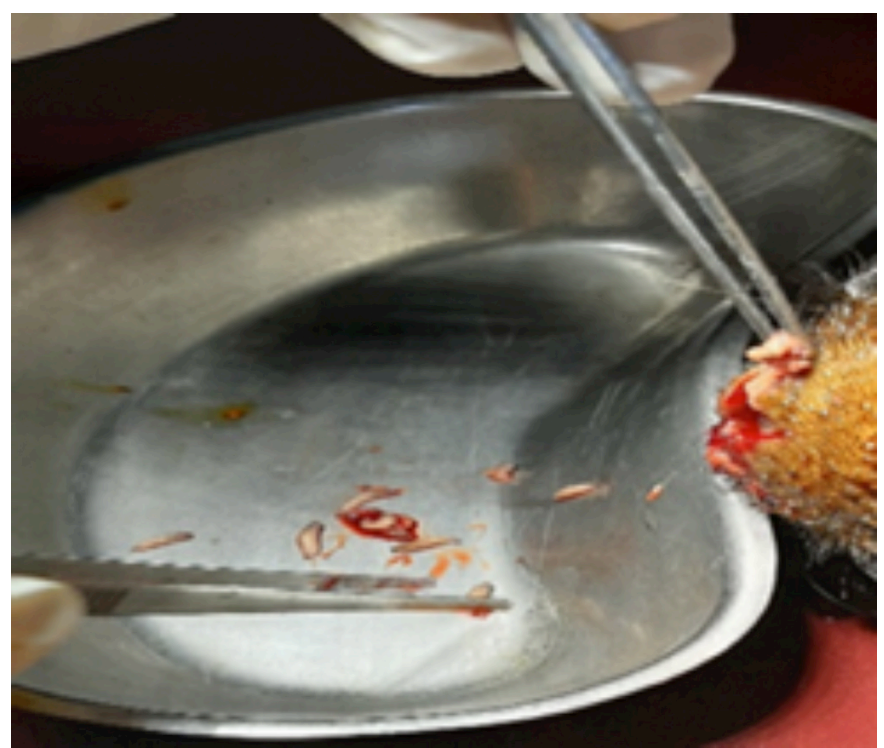

Figure 1c: Retrieval of maggots in a kidneytray.

On initial examination, a crater shaped, $4 \times 5 \mathrm{~cm}$ abscess was present on the vertex of the scalp with necrotic edges, thick pus; and multiple mobile maggots in its base (Figure 1a). Viral profile was negative for hepatitis B, C and HIV. Complete blood picture showed hemoglobin of $9 \mathrm{~g} / \mathrm{dL}$ and total lymphocyte count of $11 \times 10^{9} / \mathrm{L}$. Computed tomography scan of the brain without contrast was normal. Based on history and examination, a diagnosis of furuncular scalp myiasis was made. After informed consent for short general anesthesia, patient was shifted to the operating room. Necrotic edges of the ulcer were excised and extended in a cruciate fashion (Figure 1b). Wound was thoroughly washed with pyodine, and duly diluted with normal saline. Maggots were identified and taken out with plain dissecting forceps (Figure 1c). After clearing whole field of the maggots, petroleum jelly was applied deep inside the wound; and the wound was properly covered with sterilized bandages. Specimens of maggots were collected and sent to Zoology Department, University of Peshawar, which were later identified as Musca domestica (house fly). On follow-up visit after seven days, wound was clear of pus and maggots.

Myiasis is an embarrassing situation both for the patient and the physician. Myiasis of many organ systems have been reported, cutaneous myiasis being the most common form and presents in three different forms, i.e. furuncular, creeping (migratory), and wound (traumatic). ${ }^{1,2}$ The typical cutaneous manifestation of furuncular myiasis is red-raised area, having a central punctum, with larvae seen inside it. The most commonly documented symptoms are pruritus, pain, and movement sensation. ${ }^{1-3}$ Treatment of myiasis, after its timely diagnosis, is straight-forward and most often successful. Current treatment 
regimen includes physical removal of accessible larvae, and suffocations with petroleum-based preparations, if not accessible. This is followed by liberal irrigation of the wound with antiseptics and then adequate antibiotics cover is provided. ${ }^{4,5}$

Poor sanitation, poor socioeconomic conditions, and lack of awareness as well as education are at the heart of the problem. Statistics and policy guidelines regarding prevention of human myiasis are lacking. Currently, there is no culture of registration of such cases. Healthcare professionals should be aware of the problem and its treatment. Timely identification and treatment leads not only to symptoms resolution; but also discourages the flies causing myiasis from permanently inhibiting those areas.

\section{CONFLICT OF INTEREST:}

Authors declared no conflict of interest.

\section{AUTHORS' CONTRIBUTION:}

AK: Conception, data acquisition, interpretation, drafting, final approval.

FK: Drafting, analysis, Interpretation, critical revision, final approval.

JA HU: Conception, data acquisition, critical revision, final approval.

WA: Interpretation, critical revision, final approval.

\section{REFERENCES}

1. McGraw TA, Turiansky GW. Cutaneous myiasis. J Am Acad Dermatol 2008; 58(6):907-26. doi: 10.1016/j.jaad. 2008.03.014.

2. Francesconi F, Lupi O. Myiasis. Clin Microbiol Rev 2012;
25(1):79-105. doi: 10.1128/CMR.00010-11.

3. Caumes E, Carrière J, Guermonprez G, Bricaire F, Danis M, Gentilini M. Dermatoses associated with travel to tropical countries: A prospective study of the diagnosis and management of 269 patients presenting to a tropical disease unit. Clin Infect Dis 1995; 20(3):542-8. doi: 10.1093/clinids/20.3.542.

4. Biswas S, McNerney P. Furuncular myiasis of the scalp. Eplasty 2016; 16:ic21.

5. Sunny B, Sulthana L, James A, Sivakumar T. Maggot infestation: Various treatment modalities. J Am Coll Clin Wound Spec 2016; 8(1-3):51-53. doi: 10.1016/j.jccw. 2018.03.002.

Abdullah Khan ${ }^{1}$, Faheemullah Khan ${ }^{2}$, Jawad Ahmad', Hameed Ullah $^{1}$ and Wiqar Ahmad ${ }^{3}$

${ }^{1}$ Department of Neurosurgery, Lady Reading Hospital, Peshawar, Pakistan

${ }^{2}$ Department of Radiology, The Aga Khan University Hospital, Karachi, Pakistan

${ }^{3}$ Department of Internal Medicine, Northwest General Hospital $\&$ Research Centre, Peshawar, Pakistan

Correspondence to: Dr. Faheemullah Khan, Department of Radiology, The Aga Khan University Hospital, Karachi, Pakistan.

E-mail: islamianfellow@hotmail.com

Received: November 23, 2019; Revised: December 30, 2019; Accepted: February 10, 2020

DOI: https://doi.org/10.29271/jcpsp.2020.11.1234 